- Case Report

\title{
Intravesical Migration of an Intrauterine Contraceptive Device with Secondary Calculus Formation
}

\author{
Amit Sharma*, Mukund Andankar, Hemant Pathak \\ Department of Urology, TNMC \& BYL Nair Hospital, Mumbai, India
}

Intrauterine contraceptive devices (IUCDs) are a common form of reversible contraception owing to fewer systemic side effects and low cost, especially in a developing country like India. However, IUCDs are not without complications. Migration of a device into adjacent organs is the most morbid of all the documented complications. A patient who presents with a history that suggests loss or disappearance of an IUCD thread associated with urinary symptoms should raise suspicions that a device may have migrated into the bladder. Physicians should also be aware of possible secondary vesical calculus formation. Further radiological investigations and appropriate management are warranted. We present a case report describing the migration of an IUCD into the bladder with secondary calculus formation.

Keywords: Intrauterine Devices; Urinary Bladder; Calculus 


\section{INTRODUCTION}

Intrauterine contraceptive devices (IUCDs) are one of the most widely chosen contraceptive options with over $99 \%$ effectiveness in preventing pregnancy, a low side effect profile, and low cost. ${ }^{1)}$ However, these devices are associated with a multitude of complications including the serious risk of potential device migration into the intraperitoneal cavity and surrounding viscera. ${ }^{1)}$ The rate of transuterine perforation and migration of IUCDs into the abdominal cavity has been estimated at less than $0.1 \% .{ }^{2)}$ We report a 30 -year-old female who presented with IUCD migration into the urinary bladder with secondary calculus formation.

\section{CASE REPORT}

A 30-year-old female was admitted who had been experiencing suprapubic pain and dysuria since previous 2-3 months. There was no his-

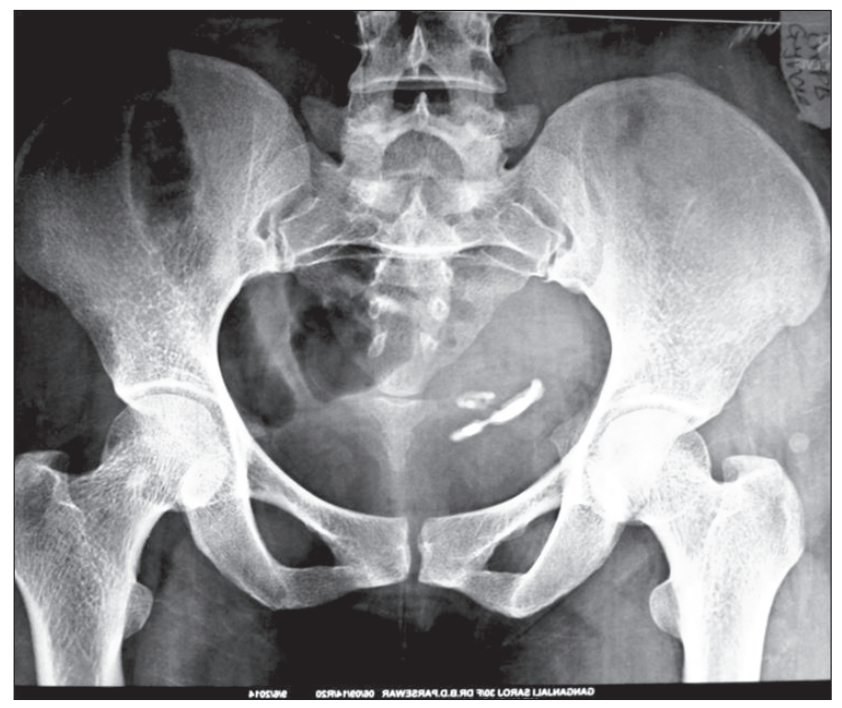

Figure 1. Kidney, ureter, and bladder radiography showing a thin, elongated, calculus-like foreign body. tory of hematuria, pyuria, lithuria, burning micturition, or fever with chills. She had no history of previous surgery or any comorbid illnesses. She was married and had one child. Copper $\mathrm{T}$ device was inserted three years prior.

She was stable at admission. Her abdomen was soft and non-tender, and the urinary bladder was not palpable. The external urethral meatus was normal. A routine vaginal examination was normal. Hematological investigations indicated that renal and liver functions were normal. Urine analysis and microscopy showed 6-8 pus cells/high power field, and there were no red blood cells.

Kidney, ureter, and bladder radiography showed a thin, elongated, T-shaped, calculus-like foreign body (Figure 1). Abdominal ultrasonography suggested that both kidneys were normal. There was a small calculus of $3 \mathrm{~mm} \times 3 \mathrm{~mm}$ size in the mid-pole of the left kidney. The urinary bladder was well distended and thin-walled, and a T-shaped echogenic focus with a curvilinear tail was observed within the blad-
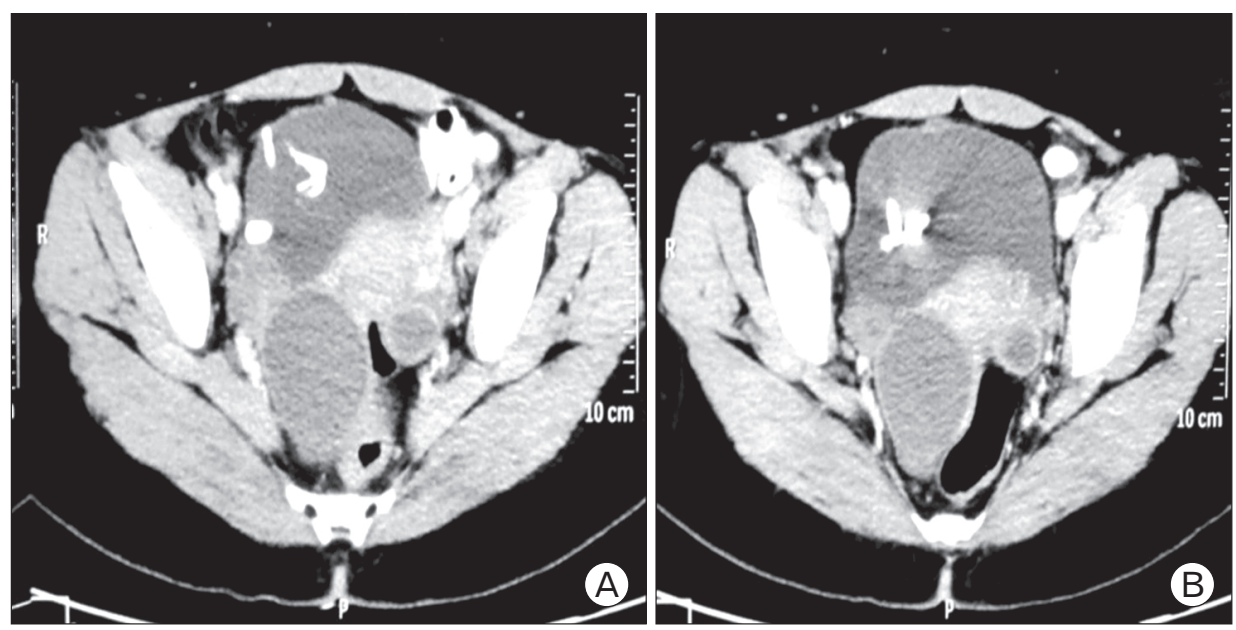

Figure 3. (A, B) Computed tomographic scan showing a distended urinary bladder with evidence of a Copper T noted in the urinary bladder with its thread encrusted. 
der, suggestive of an IUCD-Copper T device (Figure 2).

Computed tomography (CT) showed a distended urinary bladder with evidence of a Copper T noted in the urinary bladder with its thread encrusted (Figure 3). The long arm of the Copper T appeared to be impacted in the right lateral wall of the bladder and was projecting slightly beyond the surface of the urinary bladder. However, no obvious fistulous tract was noted.

Cystoscopy showed an encrusted Copper $\mathrm{T}$ in the bladder with an impacted tread in the right lateral wall of the bladder. The Copper $\mathrm{T}$ was removed with the help of double J stent removal forceps after incising the thread on the lateral wall with a Collins knife.

\section{DISCUSSION}

IUCDs are the most popular method of reversible contraception in India because of their high efficacy for fertility regulation, low-risk, lowcost, and lack of required maintenance. ${ }^{3,4)}$ They are ideal for lactating women because they do not affect the quality and composition of breast milk. ${ }^{4)}$ The possible complications associated with IUCD use are device failure to prevent contraception, painful abdominal cramps, expulsion, complete or partial uterine perforation, device migration, menstrual disturbances, increased risk of ectopic pregnancy, septic and spontaneous abortion in case of pregnancy with an IUCD, and pelvic inflammatory disease. ${ }^{4)}$

Migration of IUCDs to the rectum, sigmoid, small bowel, and peritoneum has been reported in literature. ${ }^{3)}$ The majority of the migrated and misplaced IUCDs perforate the uterus completely and remain in the pelvic cavity while some become embedded in the omentum. ${ }^{2)}$ Early closed loop devices like the Birnberg bow were associated with small bowel strangulation and were, hence, discontinued. ${ }^{2)}$ Copper coils induce marked peritoneal reactions, causing adhesion formation and bowel injury. ${ }^{2)}$ Lippes Loops and Saf-T-Coils are inert and nonmedicated, hence, they cause little biological reaction and can be quiescent for long periods. ${ }^{2)}$ Modern intraperitoneal IUCDs have low morbidity and can be left in situ. ${ }^{2)}$

There are many reports in literature that describe migration of an IUCD into the urinary bladder.5) Normally, the uterus is in an anteverted and anteflexed position and lies in close proximity to the urinary bladder; thus, there is a high propensity of migration of the IUCD into the bladder. ${ }^{5)}$ The process of transmigration of foreign bodies across organs is quite complex and difficult to understand. ${ }^{5)}$

Once the IUCD migrates to the urinary bladder, the patient will experience urinary symptoms like frequency, dysuria, hematuria, retention of urine, and fever due to infection. ${ }^{5)}$ Calculus formation occurs in long-standing cases. ${ }^{5)}$ Dietrich reported eight cases of intravesical IUCD migration in 1992 with development of urinary symptoms as early as 3 months to as late as 5 years after insertion. ${ }^{3,6)}$ The index patient was also having similar urinary complaints for three months prior to her admittance.
Patients are usually treated for urinary tract infections as the symptoms are non-specific and the diagnosis is usually delayed. ${ }^{5)}$ Hence, the importance of a good and proper history cannot be overemphasized. Simple investigations like plain radiography and ultrasound of the pelvis are enough to arrive at a diagnosis. ${ }^{5}$ A CT scan can precisely locate the migrating IUCD in the pelvic/abdominal cavity and help in planning the appropriate management. ${ }^{4)}$

Laboratory investigations usually reveal leukocytosis in infected cases, and the urine examination will reveal either pus cells or microscopic or gross hematuria. ${ }^{5)}$ Surgery is the mainstay of treatment for migrated IUCDs with calculus formation. ${ }^{5)}$ It has been suggested that misplaced and migrated IUCDs should be removed even in asymptomatic patients because of the risk of adhesion formation and damage to the surrounding structures. ${ }^{4}$

A preliminary cystoscopic examination is mandatory to chart the course of further intervention. ${ }^{5,7)}$ For mobile stones, the endoscopic methods will suffice. ${ }^{5)}$ However, if the stone is large and fixed, then open suprapubic cystolithotomy is indicated for safe and complete removal. ${ }^{5)}$

Perforation and migration into adjacent structures are known complications of IUCDs and should be kept in mind when a female with a history of IUCD insertion presents with urinary symptoms. The importance of a good history, appropriate post-insertion follow-up, and counseling of the patient cannot be overemphasized.

\section{CONFLICT OF INTEREST}

No potential conflict of interest relevant to this article was reported.

\section{REFERENCES}

1. Subramanian V, Athanasias P, Datta S, Anjum A, Croucher C. Surgical options for the retrieval of a migrated intrauterine contraceptive device. J Surg Case Rep 2013;2013:rjt072.

2. Brar R, Doddi S, Ramasamy A, Sinha P. A forgotten migrated intrauterine contraceptive device is not always innocent: a case report. Case Rep Med 2010;2010:740642.

3. Aggarwal S, Jindal RP, Deep A. Intravesical migration of intrauterine contraceptive devices with stone formation. J Family Med Prim Care 2014;3:449-51.

4. Mbamara S, Omojuwa I. An unusual presentation of perforated intrauterine contraceptive device. Ann Med Health Sci Res 2013;3:274-6.

5. Vagholkar S, Vagholkar K. Secondary vesical calculus resulting from migration of an intrauterine contraceptive device. Case Rep Obstet Gynecol 2012;2012:603193.

6. Dietrick DD, Issa MM, Kabalin JN, Bassett JB. Intravesical migration of intrauterine device. J Urol 1992;147:132-4.

7. Van Ophoven A, deKernion JB. Clinical management of foreign bodies of the genitourinary tract. J Urol 2000;164:274-87. 